\title{
Successful late non-surgical removal of intracardiac catheter fragment
}

\author{
J F REIDY, P B DEVERALL, E SOWTON \\ From the Departments of Radiology, Thoracic Surgery, and Cardiology, Guy's Hospital, London
}

SUMMARY A patient was discovered to have a retained intracardiac catheter fragment more than two years after successful aortic valve replacement. During this time the patient had had extensive investigations and several hospital admissions for suspected subacute bacterial endocarditis, as well as for episodes of supraventricular tachycardia. The catheter fragment was successfully removed via a percutaneous catheter approach despite the very long interval that had elapsed since its detachment.

Since 1964 when the first case of non-surgical removal of an intravascular catheter fragment was described ${ }^{\prime}$ there have been many accounts of successful cases of percutaneous removal and much has been written on the different techniques available. In a review on the current techniques of non-surgical removal of intravascular iatrogenic foreign bodies it was found that 220 cases of catheter emboli had been reported, with 69 cases of non-surgical removal. ${ }^{2}$ Of these, a small number of patients had had no complications during follow-up of up to six years. There were two other groups of patients who had either fatal or nonfatal complications. In both of these groups the longest period that elapsed from occurrence of emboli to death or removal of the fragment was one year, with most occurring after a much shorter period of time. No mention is made in the published reports of the relative risks of late versus early complications and also of the feasibility of late percutaneous removal of catheter fragments.

\section{Case report}

A man of 52 was referred for operation with a five year history of progressive symptoms of mixed aortic valve disease. At operation (6 February 1979) a heavily calcified aortic valve was replaced with a $25 \mathrm{~mm}$ Björk-Shiley prosthesis. There were no postoperative complications and 13 days later the patient was discharged from hospital. He remained on long-term oral anticoagulation. Three days after his discharge he was readmitted at his general practitioner's request because of irregularity of his pulse associated with tachycardia. In hospital his electrocardiogram was noted to be unchanged and as there were no further arrhythmias he was discharged a few days later. $\mathrm{He}$ remained fairly well for the next nine months and until 10 months after his operation when he was admitted to hospital with a clinical diagnosis of subacute bacterial endocarditis. He had had two episodes of feeling weak and unwell, associated with fevers, shivering, and night sweats. On examination apart from some pyrexia $\left(37.4^{\circ} \mathrm{C}\right)$ there were no abnormal findings and the aortic valve prosthesis appeared to be functioning satisfactorily. A further admission two months later (9 February 1981) was necessary for a similar episode. On both these admissions multiple blood cultures were taken, but no significant growth was found (nine series of blood cultures showed no growth with the exception of one bottle out of a series of three which showed a growth of Staph. albus which was considered to be a contaminant).

When the patient was again seen in out-patients in May 1981 with symptoms suggestive of subacute bacterial endocarditis it was noted on a chest $x$-ray that there was a very faint outline of catheter seen in the superior vena cava and extending into the right atrium. Review of previous postoperative films showed that this faint shadow had been present on other films and since the early postoperative period (Fig. 1).

He was admitted on 8 June 1981 for attempted percutaneous removal of the catheter fragment before an elective thoracotomy. Under local anaesthesia a $9 \mathrm{~F}$ 
gauge introducer sheath was introduced via the percutaneous approach into the right femoral vein. Through this various retrieval systems could be passed to snare the free end of the catheter. After several attempts under fluoroscopy the free end of the catheter in the superior vena cava was successfully snared using a basket retrieval kit. ${ }^{\star}$ The catheter fragment was withdrawn into the end of the sheath and the sheath and catheter fragment were all pulled out together. There were no complications. The catheter

^Dotter intravascular retriever set. William Cook, Europe

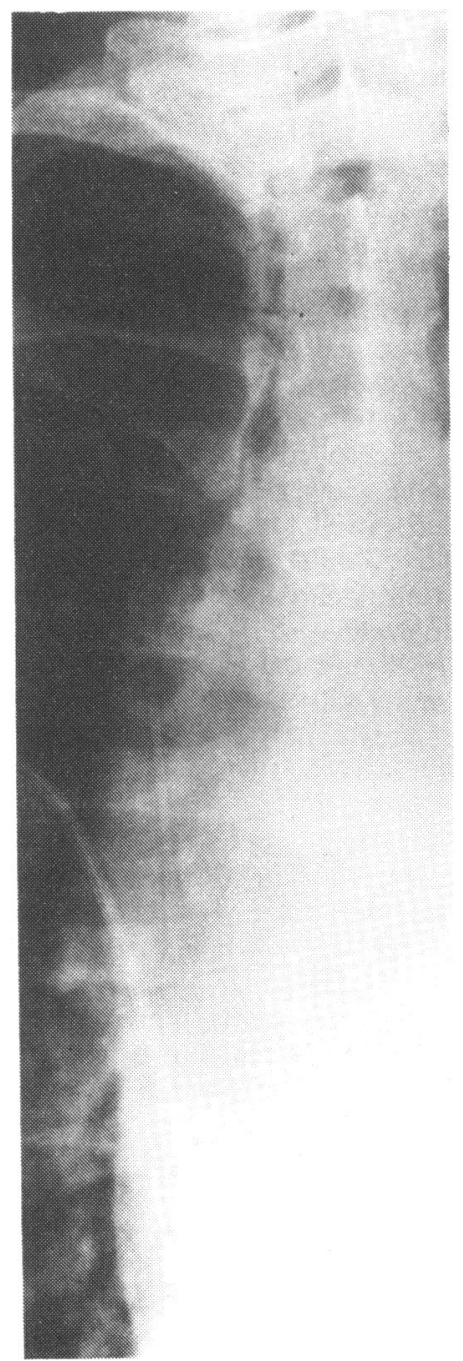

Fig. 1 Detail of a chest radiograph. Only the portion of the catheter in the region of the superior vena cava is clearly visible. (The thicker and more obvious, laterally placed linear shadow is a monitoring cable on the patient's chest wall.)

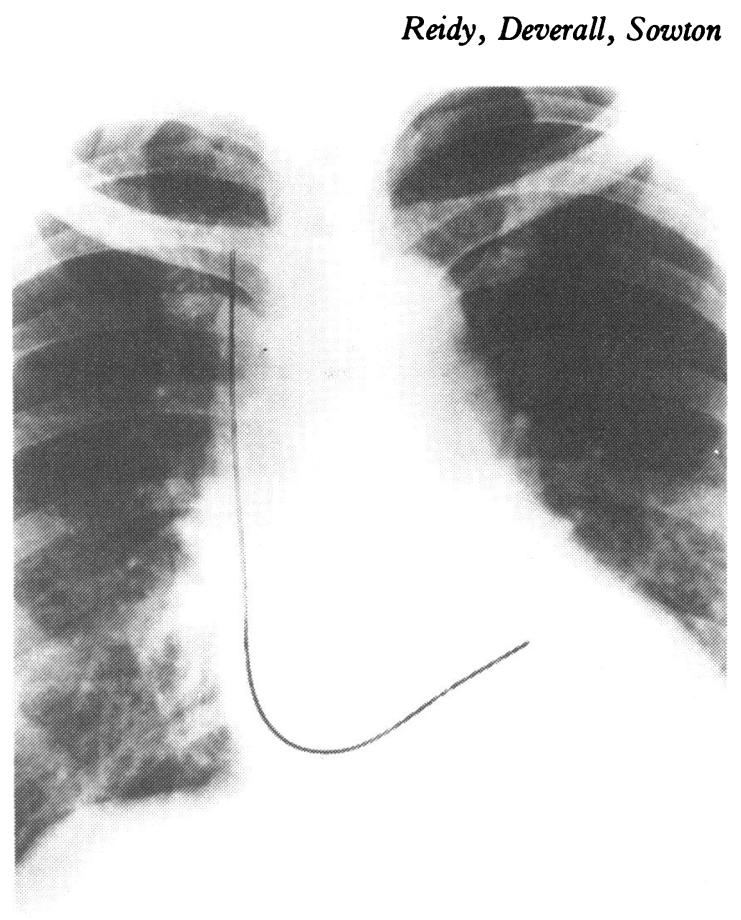

Fig. 2 The catheter fragment after its removal is shown superimposed on the chest radiograph. The portion that was in the right atrium and ventricle was not seen on chest radiographs before removal.

fragment, which measured $27 \mathrm{~cm}$, was immediately sent for culture but no growth was obtained from it. The catheter fragment proved to be from a central venous pressure line system. For the removal the patient was put on antibiotic cover and he remained on his oral anticoagulants. He was discharged the following day and when seen one month later felt very well and had had no further episodes of fever. The catheter fragment is shown (Fig. 2) attached to the chest $x$-ray and overlying its radiographic position. It appears much larger than on the most penetrated and optimal chest radiograph.

\section{Discussion}

The morbidity and mortality associated with retained intravascular catheter fragments are very difficult to assess. Undoubtedly the reported cases underestimate the true incidence and early deaths usually caused by arrhythmias could easily be ascribed to other causes. Of 16 deaths resulting from catheter fragment embolisation, five occurred at two days or less. ${ }^{2}$ In the same review seven of the 16 deaths occurred over 40 days after the embolism, two occurring one year later. Thus, other series tend to underestimate the problem by having only a short term follow-up period. In addition, it is often difficult conclusively to implicate the 
embolised fragment as the main cause of death. It is therefore not surprising that estimates vary considerably. In their review, Fisher and Ferreyo ${ }^{2}$ cite a $71 \%$ risk of death or serious complications. In arriving at this figure they do not include cases followed up for less than four months which have no complications. Other figures have varied from a mortality of $60 \%{ }^{3}$ to $24 \% .{ }^{4}$ It is generally accepted that the risks resulting from an intracardiac embolism are greater than one outside the heart.

Arrhythmias tend to occur in the early period after intracardiac catheter embolism, while clot emboli and infective episodes tend to occur later. Our patient, after an initial arrhythmic episode, remained well for about 10 months. He then presented with several episodes that clinically appeared as subacute bacterial endocarditis. On all these occasions multiple blood cultures were negative. The diagnosis was only established when the faint linear density of the fragment was seen overlying the superior vena cava on a well penetrated chest $x$-ray. There were no episodes to suggest pulmonary emboli but the patient had been on long term anticoagulation throughout.

The approximate time interval between the embolism occurring and the removal of the fragment was two years and four months. In view of this time interval it was anticipated that the catheter might be attached to the wall of the vena cava and/or the right atrium and that this would make percutaneous removal impossible or difficult. In fact once the free end of the catheter was snared there was no difficulty and no force was required to withdraw it.

\section{References}

1 Thomas J, Sinclair-Smith B, Bloomfield D, Davachi A. Non-surgical retrieval of a broken segment of steel spring guide from the right and inferior vena cava. Circulation 1964; 30: 106-8.

2 Fisher RG, Ferreyro R. Evaluation of current techniques for nonsurgical removal of intravascular iatrogenic foreign bodies. AfR 1978; 130: 541-8.

3 Benhardt LC, Wegner GP, Mendenhall JT. Intravenous catheter embolization to the pulmonary artery. Chest 1970; 57: 329-32.

4 Richardson JD, Grover FL, Trinkle JK. Intravenous catheter emboli; experience with 20 cases and collective review. Am $\mathcal{F}$ Surg 1974; 128: 722-7.

Requests for reprints to Dr J F Reidy, Department of Radiology, Guy's Hospital, London SE1 9RT. 